concealing females behind the inlet screen, and adding the test substances to the air intake. Not only would this provide a direct route to metarchon formulation, but it would throw considerable light on the olfactory process itself.

I thank the Canada Departments of Agriculture and Forestry for grants.

\section{British Columbia Research Council,} Vancouver 8, Canada.

1 Wright, R. H., Nature, 204, 603 (1984).

Wright, R. H., Nature, 204, 121 (1964).

- Kellogg, F. E., and Wright, H. H., Canad, Ent., 94, 486 (1962).

\section{Damage to Polythene by Aquatic Moths}

Reconds of demage to polythene by insects are now fairly frequent. The larva of the common household pest Hofmannophila pseudospretella Steinton (the Brown House or False Clothes moth) is known to eat its way through many man-made products, including polythene, polystyrene, and nylon.

Recently the larvae of an aquatic lepidopteran species were found damaging the polythene linings of ponds (1,000 geuge, approx. $0.25 \mathrm{~mm}$ thick). The damage to the inner lining of the pond was caused by the larva of the moth eating holes through the polythene to the rough paper outer-lining. The larvae then attached themselves to this outer-lining paper before pupating. After the moths emerged the pupal cases were soon washed away, leaving holes of $1-3 \mathrm{~mm}$ diameter. This demage occurred both in a greenhouse where the pond was a wood-fremed structure with a paper underlay and a polythene lining, and out-of-doors where soil was underneath the polythene. All the holes were at or just above water-level.

It is possible that the very smooth polythene failed to provide a suitable surface on which the larvae could anchor themselves before pupation and that they therefore chewed through to a rougher surface. Perhaps if the polythene surface was rougher the larvae would attach themselves without burrowing through it. The damage was first noticed in 1964 although the polythene had been in use for 7-8 years.

Specific determination of the moths was not possible in the sample examined, as all the pupal cases were empty. However, it is certain that the moths concerned were a species of Hydrocampinae (Pyralidae) and almost certain that the species was Nymphula nympheata Linnaeus, the Brown China-Mark moth.

Paul E. S. Whalley

British Museum (Natural History), London, S.W.7.

\section{MICROBIOLOGY}

\section{Variation in the Chemical Composition of the Cell Walls of Bacillus subtilis during Growth in Different Media}

DuRINe recent years the chemical composition of the cell wall has been used as an aid in the classification of micro-organisms ${ }^{1}$. Although differences in the cormposition of the cell wall have been demonstrated during growth of organisms with: (a) variable morphological forms ${ }^{2}$; (b) complex auxotrophic requirements ${ }^{3}$; (c) addition of D-amino-ecids 4 , many investigations have not considered the variationg which occur in the amino-acid and aminosugar content of more stable stains of bacteria during the growth cycle and in response to different growth media. The results presented here demonstrate that the amino-acid and amino-sugar content of the cell wall of Bacillus subtilis varies not only with growth but is also significantly influenced by the composition of the growth medium. Furthermore, galactosamine, one of the major components of the cell wall of the highly transformable strain $B$. subtilus $168 \mathrm{I}^{-} \mathrm{C}^{+}$, is consistently decreased when this strain is grown under conditions which do not permit the development of the capacity to bind deoxyribonucleic acid (competence). A marked decrease in galactosamine is also observed in three poorly transformable strains of B. subtilis.

$B$. subtilis $168 I-C^{+}$was grown for various intervals at $37^{\circ} \mathrm{C}$ with vigorous aeration in 800 -c.c. cultures contained in 2-1. flasks. Three media were used: $(A)$ the standard growth medium containing 0.5 per cent glucose, $0.005 \mathrm{M}$ $\mathrm{MgSO}_{4}, 50 \mu \mathrm{g} / \mathrm{ml}$. L-tryptophan, and 0.02 per cent casein hydrolysate ${ }^{5} ;(B)$ the previous medium supplemented with $0 \cdot 4$ per cent casein hydrolysate; $(C)$ 'Pen Assay' broth (Difoo). B. subtilis 168 does not sporulate in these media under the experimental conditions used. The organisms were collected by centrifugation, washed and disrupted by mechanical disintegration ${ }^{6}$. Samples of cell walls were dried over phosphorus pentoxide, hydrolysed at $105^{\circ} \pm 1^{\circ} \mathrm{C}$ for $11 \mathrm{~h}$ in $4 \mathrm{~N}$ hydrochloric acid, and evaporated in vacuo to remove the hydrochloric acid. The amino-acid and amino-sugar content was determined on the Beckman Spinco amino-acid analyser ${ }^{6}$.

Previous experiments have shown that logarithmic growth in medium $A$ occurs $1.5-5 \mathrm{~h}$ after incubation ${ }^{5,7}$. The culture then remains in a stationary period for $3 \mathrm{~h}$ before a marked decrease in the number of viable organ. isms occurs. As shown in Table 1 the major amino-acids and amino-sugars in the cell wall of $B$. subtilis are alanine, glutamic acid, diaminopimelic acid, muramic acid, glucosamine, and galactosamine. During the logarithmic and early stationary phases of growth $(1 \cdot 5-8 \mathrm{~h})$ the amounts of glutamic acid, diaminopimelic acid, and glucosamine do not vary beyond the limit of the accuracy of this method ( \pm 5 per cent on repeated analyses of the major components in the same sample of cell walls). On the other hand, the content of alanine within the cell wall increased during incubation. A consistent decrease in muramic acid was observed after $5 \mathrm{~h}$ of growth. The highest concentration of galactosamine occurred after $5 \mathrm{~h}$ of incubation which coincided with the time that the population developed maximal competence. Since the galactosamine is confined to the teichoic acid fraction of the cell walls ${ }^{8}$, this two-fold increase in galactosamine might significantly alter the structure of the cell wall. After $24 \mathrm{~h}$ of incubation there was an increase in all the major components except galactosamine. The small differences in the minor components (aspartic acid to arginine, Table 1) are not significant.

Previous investigations have demonstrated that growth in nutrient media such as media $B$ and $C$ inhibit the development of competence ${ }^{5}$. As shown in Table 2, there is an increase in alanine and a decrease in galactosamine under these conditions of growth. This decrease in galactosamine was observed after both 3 and 5 h of incubation in 'Pen Assay' broth.

Two additional poorly transformable strains of $B$. sub. tilis were examined. B. subtilis 23 is en excellent donor strain in transformation experiments, but a very poor recipient. $B$. subtilis $H$ is essentially not transformable. As shown in Table 3 , the most outstanding difference was in the galactosamine content of the cell wall. At present it is not known whether galactosamine is an integral part of the teichoic acid polymer or is in another polymer which is fortuitously isolated with the teichoic acid fraction during purification. Nevertheless, it is clear that galactosamine varies during the growth cycle and is decreased when the transformable strain is grown in more complex media. In addition, the concentration of galactosamine is markedly decreased in the two poorly transformable strains used in this work and in a poorly transformable mutant of $B$. subtilis 168 (ref. 6). 